El Guiniguada • Revista de investigaciones y experiencias en Ciencias de la Educación

\title{
CREACIÓN DE PERIÓdICOS EN LÍNEA CON PAPAR.LI COMO RECURSO DIDÁCTICO E INNOVADOR EN LA UNED
}

\section{CREATING ONLINE NEWSPAPERS WITH PAPAR.LI AS A TEACHING AND INNOVATING RESOURCE IN THE UNED}

\author{
José Ramón Carriazo Ruiz \\ Elisabeth Fernández Martín \\ Yanira Navarro Marrero \\ UNED (Universidad Nacional de Educación a Distancia)
}

Recibido: 12/04/2015/Aceptado: 28/04/2015

\section{RESUMEN}

La herramienta Paper.li permite la creación de periódicos en línea, por lo que se convierte en un recurso útil e innovador en el contexto de la enseñanza y el aprendizaje a distancia con adultos, en particular en la UNED. Las publicaciones compartidas en las redes sociales, principalmente en Twitter y Facebook, y que han de estar necesariamente relacionadas con las asignaturas impartidas por el docente y cursadas por los estudiantes, constituyen el material informativo del periódico digital. Los titulares, divididos y organizados en categorías, se editan de modo colaborativo; generando así espacios para el diálogo en un atractivo formato web.

Palabras clave: editor, herramienta, innovación, lectura digital, periódico en línea.

\section{ABSTRACT}

The Paper.li tool allows you to create online newspapers, which makes it a useful and innovating resource in the context of teaching and distance learning with adults, especially in the UNED. Online articles shared in social media, mainly Twitter and Facebook, and they necessarily have to be related to the subjects imparted by the 
teacher and studied by student, creates the informative content of these digital newspapers. The headlines, divided and organized by categories, are edited collaboratively and thus generate talking points in an attractive web format.

Keywords: editor, tool, innovation, digital reading, online newspaper.

\section{INTRODUCCIÓN}

El desarrollo de la Web 2.0 o Web social ha propiciado el surgimiento de una serie de plataformas y herramientas que permiten a los usuarios ser productores activos de información, frente a lo que ocurría en la Web 1.0, donde solo podían ser consumidores pasivos de información. Por otro lado, mientras en la Web 1.0 el usuario tiene que ir a buscar la información, en la Web 2.0 al usuario le llega la información porque previamente ha establecido filtros para que ello suceda.

El modelo educativo que integra las Tecnologías de la Información y la Comunicación (TIC) en el proceso de enseñanza y aprendizaje se ha venido a llamar Educación 2.0, como apunta Toro (2010, 4); a día de hoy es una realidad que no puede pasarnos desapercibida y a la que no podemos dar la espalda.

El avance imparable de Internet como medio de comunicación de masas ha provocado cambios de envergadura en los modos de comunicación entre los equipos docentes y el alumnado. Según Mancera $(2011,54)$, «la renovación constante de los contenidos puede provocar en el estudiante la sensación de encontrarse ante discursos en continua evolución, o en cierta medida inacabados. Para ordenar, jerarquizar, orientar y dinamizar la captación de noticias y promover el espíritu crítico ante las novedades recogidas en las redes sociales, se han diseñado «periódicos personalizados» como el denominado fishwrap 'envoltorio de pescado'».

La metodología de recopilación mediante gráficos de noticias de interés se denomina «el prisma conversacional» (en inglés: the conversation prism). Las dos principales herramientas para su aplicación son Flipboard (https:// flipboard.com/), basado en tecnología para móviles y disponible para iOS, Windows 8 y Android, y Paper.li (bttp://paper:li/). 


\section{Imagen 1. Representación del prisma conversacional}

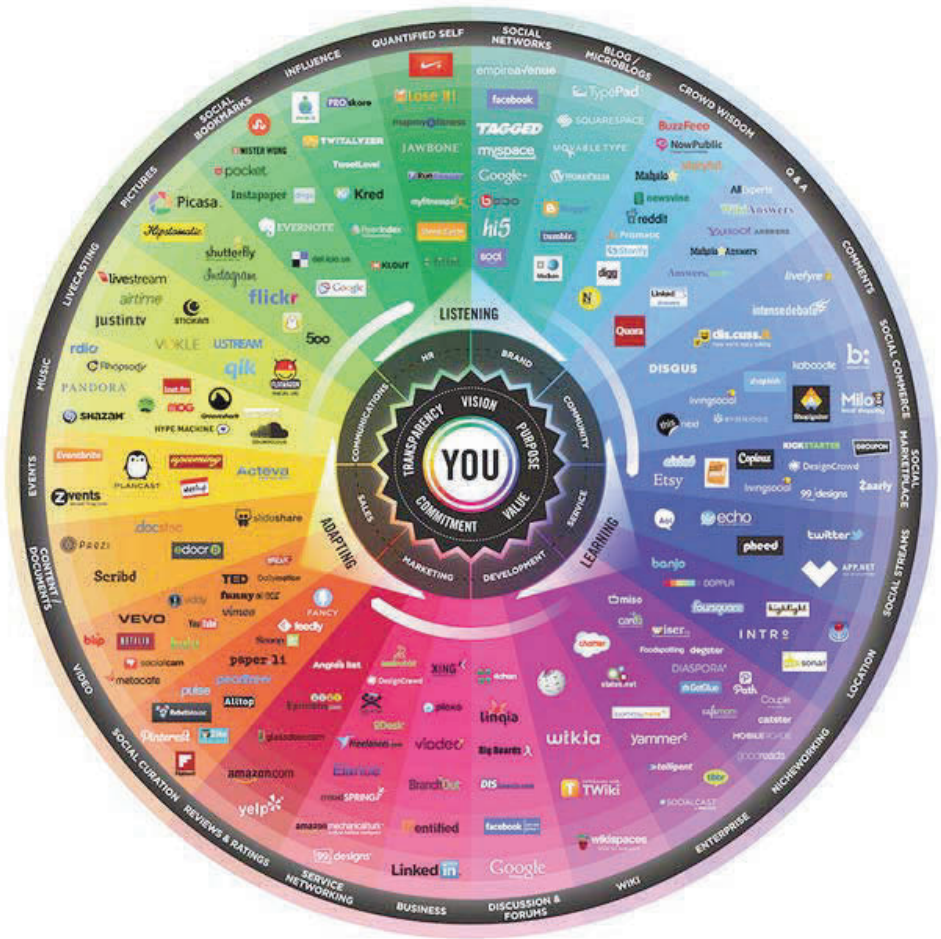

Disponible en bttp:// mwn.conversationprism.com

Aunque en un principio los blogs, las wikis, los podcasts, las redes sociales, etc. no se crearon con fines didácticos o pedagógicos, «los docentes de diferentes asignaturas y niveles educativos las usan como un recurso educativo más» (Araujo, 2012). Dentro de las nuevas tecnologías que el alumnado emplea como herramientas de comunicación habituales se encuentran, como señalan Torres y Delgado (2009) Twitter (bttps:/ / twitter.com/), que es una plataforma de microblogging; y Facebook (https:/ / www.facebook. $\mathrm{com} /$ ), que es una red social. Ambas cuentan con millones de usuarios y una amplia penetración en la comunidad universitaria.

A través de una cuenta de Twitter o Facebook se puede acceder a Paper.li que es una herramienta que permite filtrar, organizar y compartir información en formato digital (artículos, fotografías, imágenes, vídeos, etc.) que se encuentra en los medios sociales para editar el periódico digital con aquellos contenidos relevantes para un determinado tipo de audiencia. Según los creadores de Paper.li, esta es la forma en la que 
se accederá a la información en el futuro, donde compartir información no será una práctica exclusiva del periodista.

Si las nuevas tecnologías son parte fundamental de las vidas cotidianas de millones de estudiantes a la hora de comunicarse y relacionarse con amigos y familiares, nos parece un motivo más que válido para usarlas e integrarlas de modo natural en el proceso de enseñanza y aprendizaje, donde el diálogo bidireccional se establece entre el docente y el alumnado. Los nuevos canales 2.0 se convierten en unos perfectos aliados de la universidad para crear y compartir periódicos con contenidos informativos de interés de las asignaturas que imparten los docentes y que cursan los estudiantes: Paper.li nos ayuda a lograr este objetivo. Estratégicamente, los seguidores de estas publicaciones, sean miembros de la comunidad universitaria o no, contribuyen en la difusión y visibilidad de la enseñanza y el aprendizaje significativos que se generan en la UNED en un entorno virtual, que no deja de ser auténtico y real.

\section{DESCRIPCIÓN DEL PROYECTO}

La UNED, en su búsqueda de la excelencia académica y de la renovación de las metodologías docentes, ha convocado en los últimos años Redes de Investigación para la Innovación Docente con el fin de potenciar la capacidad del profesorado para desarrollar e implementar en sus asignaturas nuevas ideas acordes al Espacio Europeo de Educación Superior (en adelante EEES).

En el seno de la VIII Convocatoria de Redes de Investigación para la Innovación Docente: desarrollo de proyectos para la mejora de la calidad docente en el EEES del curso académico 20142015 se inserta la propuesta «Incorporación a la metodología docente de la herramienta Paper.li para la creación de publicaciones periódicas relacionadas con la temática de las asignaturas impartidas», coordinada por los profesores José Ramón Carriazo Ruiz y Nuria Polo Cano. En concreto, esta iniciativa se vincula a la línea temática de proyectos que favorecen la incorporación de la metodología docente de propuestas didácticas innovadoras, diferentes a las utilizadas tradicionalmente; valorándose positivamente su aplicación conjunta en diferentes titulaciones de forma simultánea.

Ya en el curso académico 2013-2014 se llevaron a cabo dos experiencias piloto ${ }^{1}$ con alumnado de la UNED matriculado en Madrid: una en la línea de compartir noticias de Twitter y Facebook con los alumnos de Comunicación Oraly Escrita en Lengua Española, asignatura básica en el Grado de Estudios Ingleses en el Centro Asociado de la UNED de Madrid “Gregorio Marañón”; y otra mediante la creación de un semanario

1 Esta iniciativa surge de la mano de José Ramón Carriazo Ruiz, siguiendo las directrices del curso Redes sociales: usos para docentes, cursado por aquel el 12 de diciembre de 2014 en el Instituto Universitario de Educación a Distancia (en adelante IUED). 
con Paper.li para recopilar noticias de Twitter con los alumnos de Sociolingüistica del español, asignatura optativa de segundo ciclo de la Licenciatura en Filología Hispánica, impartida en la sede central de la UNED en Madrid.

Esta octava convocatoria de Redes de Investigación para la Innovación Docente [...] es crucial, puesto que arranca en un momento en que todas las titulaciones acaban de adaptarse al EEES. Su objetivo principal, conforme a las perspectivas de futuro de la UNED, es potenciar proyectos que persigan una mejora de la calidad docente en los estudios recién implantados. Por otro lado, pretende facilitar su desarrollo a lo largo del curso académico 2014-2015, permitiendo que su diseño se realice por anticipado al igual que se hace con la preparación de las propias asignaturas.

La creación de periódicos semanales con Paper.li en la UNED tiene un carácter interdisciplinar al estar representadas diferentes facultades (la Facultad de Filología y la Facultad de Psicología), distintas titulaciones (el Grado en Lengua y Literatura españolas, el Grado en Estudios Ingleses y el Grado en Psicología) así como diversas asignaturas: en el primer cuatrimestre Comunicación Oral y Escrita en Español I y Comunicación Oral y Escrita en Lengua Española I; en el segundo cuatrimestre Comunicación Oral y Escrita en Español II, Comunicación Oral y Escrita en Lengua Española II, Psicología del Aprendizaje, Psicología de la Emoción y Evaluación en Psicología Clínica. Es una materia anual Lengua Extranjera: Inglés, que se imparte en el Curso de Acceso Directo (en adelante CAD) del Centro Asociado de la UNED de Lanzarote.

Es importante destacar el carácter intercampus de esta red, pues en este momento están representados diversos campus y centros asociados (en adelante C.A.) de la UNED, tanto dentro como fuera de la península: el Campus de Madrid (C.A. de Madrid "Gregorio Marañón"), el Campus del Sureste (C. A. de Talavera de la Reina), el Campus del Norte (C.A. de La Rioja y C.A. de Bergara) y el Campus de Canarias (C.A. de Lanzarote).

Se desprenden dos perfiles de alumnos potenciales que se sumarán al proyecto: el universitario, con los alumnos de Grados, y el que podríamos denominar preuniversitario, los alumnos de CAD. En cuanto al número de estudiantes matriculados, son más de 400 en Comunicación oral y escrita en lengua española I y Comunicación Oral y Escrita en Lengua Española I en el C.A. de Madrid "Gregorio Marañón”, aproximadamente 20 en las asignaturas antes enunciadas en el C.A. de Talavera de la Reina, casi 300 en Psicología del Aprendiraje, Psicología de la Emoción y Evaluación en Psicología Clínica en el C.A. de Bergara y cerca de 60 alumnos de nuevo acceso de la UNED en Lengua Extranjera: Inglés en el C.A. de Lanzarote.

2 En La Bitácora de José-Ramón Carriazo, en los archivos de categoría paper.li (bttp:/ /jrcarriazo.hol.es) blog/category/paper-li/), se pueden consultar las entregas, así como los objetivos, logros y resultados del proyecto. 


\section{DesarRollo Del PROYECTO: CREACIÓN DE LOS PERIÓDICOS DIGITALES Y PRIMEROS RESULTADOS}

En el primer cuatrimestre, los profesores-tutores José Ramón Carriazo Ruiz, del C.A. de Madrid “Gregorio Marañón”, Elisabeth Fernández Martín, del C.A. de Talavera de la Reina y Yanira Navarro Marrero, del C.A. de Lanzarote, han editado los siguientes periódicos ${ }^{2}$ :

Comunicación oral y escrita en lengua española I. El periódico de los estudiantes de

COELEI y COEEI en el Centro Asociado de la UNED de Madrid-Gregorio Marañón

\section{Imagen 2. Capturas de pantalla de la edición del} periódico del día 6 de febrero de 2015
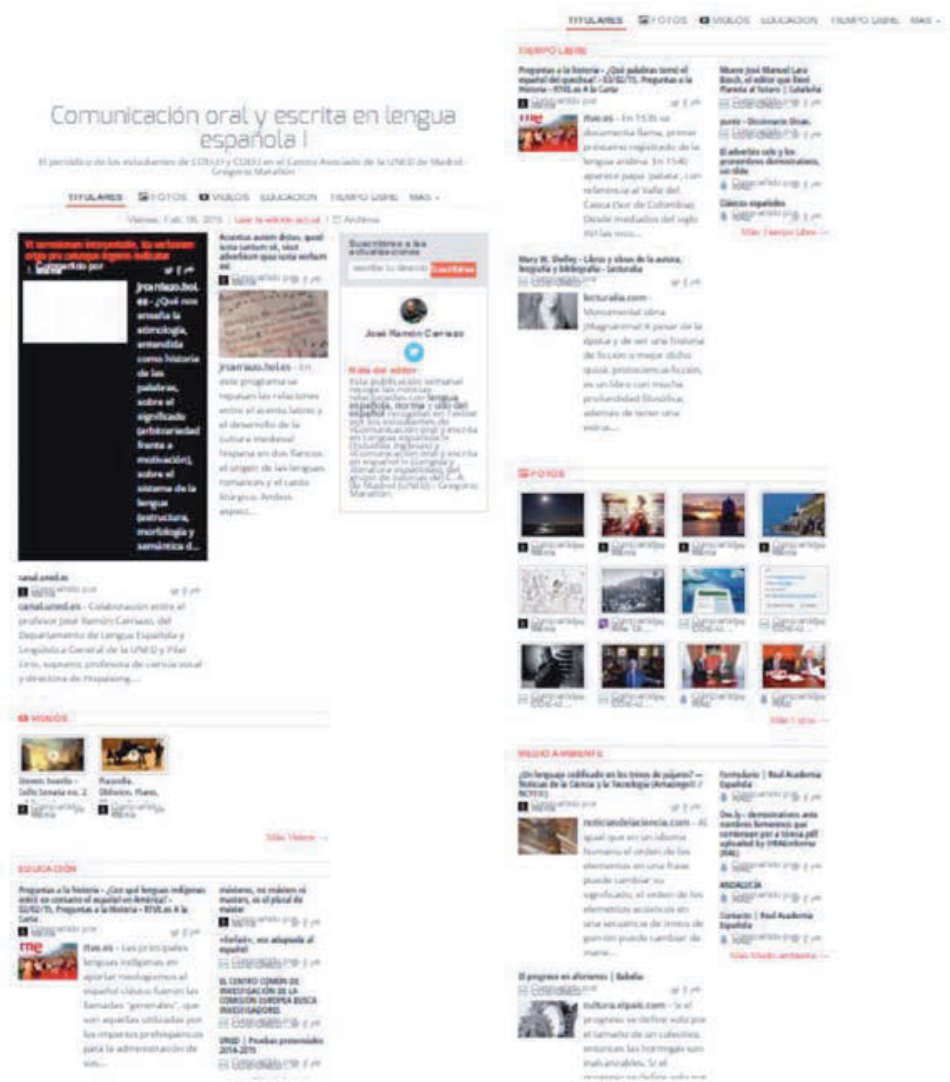

Disponible en https://paper.li/carriazojr/1412337154?edition_id= 40f1d910-adcd-11e4-9040-0cc47a0d164b 
Comunicación oral y escrita COE. El periódico de los estudiantes de COELE y COEE de la UNED (Centro Asociado de Talavera de la Reina)

\section{Imagen 3. capturas de pantalla de la edición del periódico del día 6 de febrero de 2015}

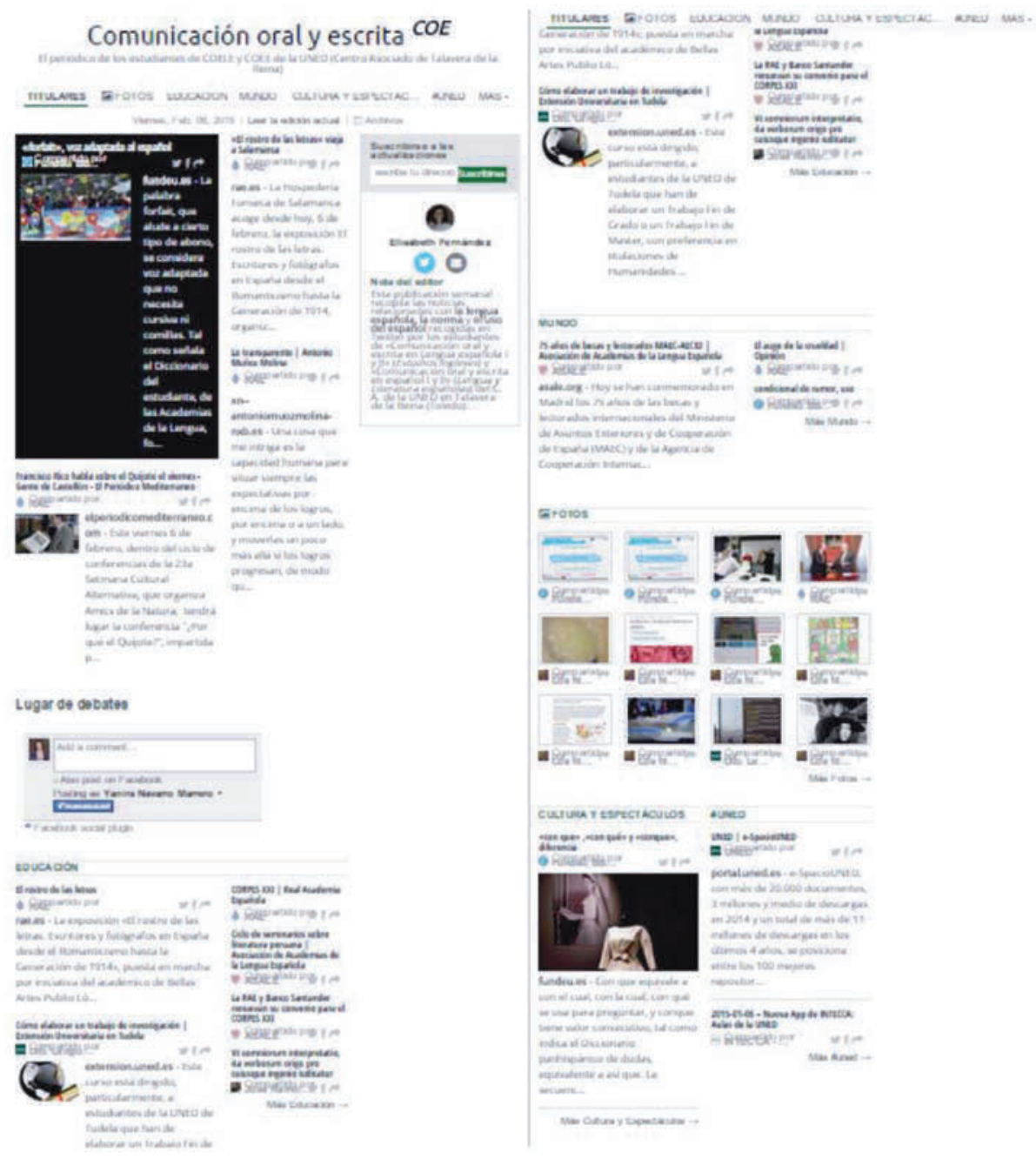

Disponible en http://paper.li/Elisabeth_FM/1414957567?edition_id= 193b380-adf6-11e4-a9d2-0cc47a0d15fd 
José Ramón Carriazo Ruiz, Elisabeth Fernández Martín, Yanira Navarro Marrero Creación de periódicos en línea con papar.li como recurso didáctico e innovador en la UNED

Lengua Extranjera: Inglés (CAD). Periódico digital colectivo de la asignatura Lengua Extranjera: Inglés (Curso de Acceso Directo) del Centro Asociado de la UNED de Lanzarote

\section{Imagen 4. Captura de pantalla de la edición del periódico del día 29 de enero de 2015}

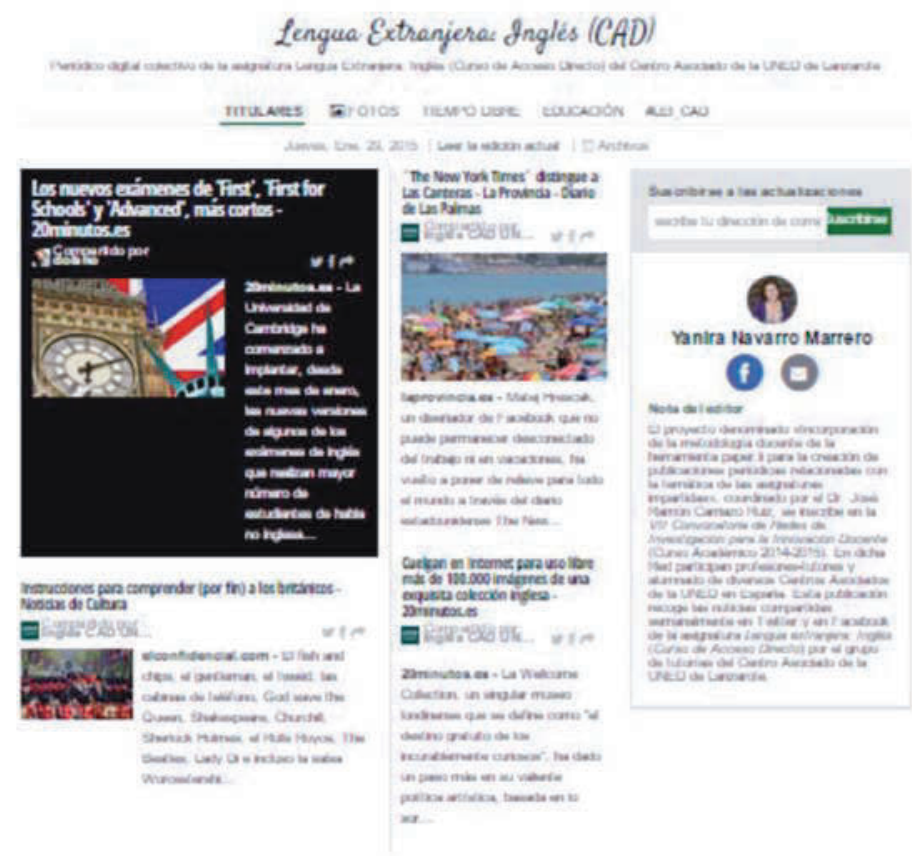

Lugar de debates

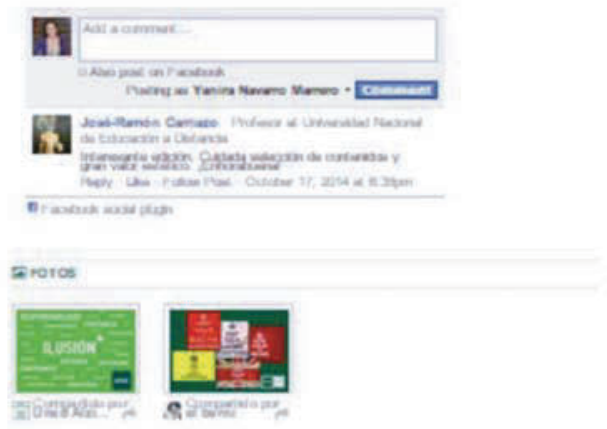

Disponible en http://paper.li/f-1413021131?edition_id=eb862b00-a748-11e4-9040-0cc47a0d164b 
La forma más sencilla de registrarse en Paper.li es a través de una cuenta de Twitter o Facebook, aunque también se puede entrar a través de un correo electrónico. Una vez registrados en esta herramienta, es posible crear tantos periódicos como se quiera de modo gratuito ${ }^{3}$.

\section{Imagen 5. Página principal de Paper.li: http://paper.li/ \\ Create your online newspaper in minutes.}
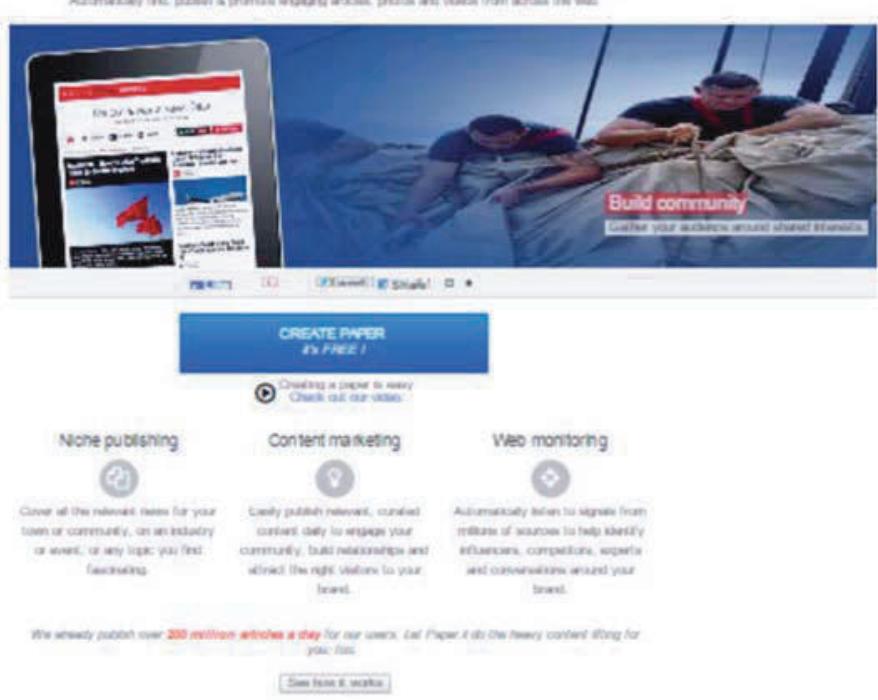

What our users are saying
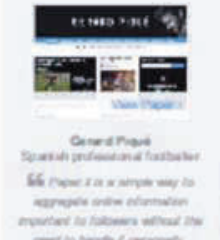

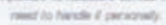
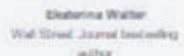

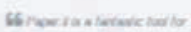

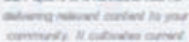

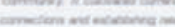

men

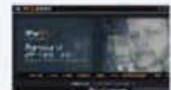

stcen kas

coomines Mata

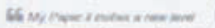

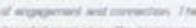

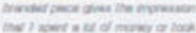

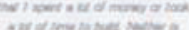

ine Mererales ation

tonele Notere

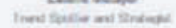

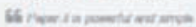

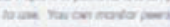

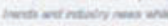

oren ins

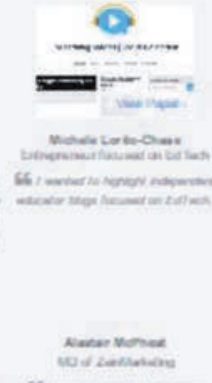

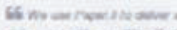

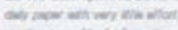

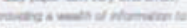

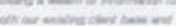

3 En la versión de pago se ofrecen más funciones, pero no nos detendremos aquí a enumerarlas. 
El periódico se crea en dos simples pasos: lo primero es ponerle un título (que se puede modificar más adelante si se desea) y lo segundo es elegir la temática sobre la que versará el periódico. Su contenido se determinará a través de una palabra clave, etiqueta o tema de interés, a partir de un nombre o una lista de Twitter o bien usando un canal RSS.

Una vez creado el periódico, se puede editar a partir de un panel de ajustes que nos permitirá realizar diferentes funciones: establecer la frecuencia de publicación; redactar una nota de editor; añadir, editar o eliminar fuentes de contenido y establecer filtros (escoger el idioma de las fuentes, elegir las secciones del periódico y bloquear sitios web o personas); modificar el aspecto del periódico (la tipografía y el fondo); configurar la promoción automática del periódico para crear audiencia en los canales sociales (Twitter, Facebook o LinkedIn) o a partir de listas de distribución, consultar las estadísticas de seguidores y visitas al periódico, etc.

El periódico está dividido en secciones, aquellas que se seleccionaron en el apartado de contenidos, y tan solo se muestran aquellas para las que se ha encontrado contenido en cada actualización. La primera sección es la de los titulares y al acceder a otras secciones se muestran los artículos que se incluyen en cada una ellas. Cada artículo se presenta como un pequeño párrafo y debajo de cada artículo se indica quién lo ha compartido.

A la hora de dotar de contenido al periódico es recomendable establecer filtros y reducir el número de fuentes, de modo que la contribución no sea tan amplia que desborde ni tan específica que no se encuentren artículos que publicar.

Se pueden seleccionar los periódicos de Paper.li de otros editores que se desean seguir y estos aparecerán en el apartado de Mis favoritos, en la sección de Newsstand. Entre otras funciones, aquí se muestran los periódicos de las personas que seguimos en Twitter o de los amigos que tengamos en Facebook, dependiendo de si hemos creado la cuenta de Paper.li con una u otra.

En lo que respecta a los primeros resultados del proyecto, hay que destacar la buena acogida que han tenido los periódicos por parte del alumnado, los comentarios positivos que han recibido, así como el seguimiento que se está haciendo de ellos. Aunque aparentemente el número de personas suscritas al periódico no sea muy alto, como se ve en el gráfico adjunto (Imagen 6), sí lo es, sin embargo, el número de visitas, que no deja de crecer en la actualidad: 838 en el caso del periódico del CA de Madrid, 213 en el de Lanzarote y 267 para el de Talavera de la Reina. Esta disparidad entre unos datos y otros se explica por el hecho de que no es necesario estar suscrito al periódico digital para poder acceder a sus contenidos; además, semanalmente y cada vez que se genera una nueva entrega, los profesores-editores enviamos un recordatorio a través de Twitter y Facebook con el enlace a la publicación. Así, por ejemplo, en el caso del periódico de Talavera de la Reina los recordatorios semanales de las 18 entregas realizadas por el momento han tenido un alcance de 582 visualizaciones 
solamente en la red social Facebook. Dicho alcance es bastante significativo, especialmente, si se tiene en cuenta el número de alumnos que hay en las asignaturas inscritas en el proyecto del CA de Talavera.

No obstante, a pesar de que los datos son muy halagüeños, debe tenerse en cuenta también que los periódicos en Paper.li son de carácter público. Eso quiere decir que permiten entrar en contacto con un público mucho mayor que el de nuestro propio alumnado, de ahí que sea imposible discernir cuáles son los datos de actividad que corresponden realmente a nuestros alumnos y cuáles no. Somos conscientes, por tanto, de que cualquier resultado estadístico que se realice debe ser matizado $\mathrm{o}$, al menos, interpretado con limitaciones.

Por otra parte, este carácter divulgativo y abierto de Paper.li nos está ofreciendo algunas considerables ventajas que no se habían contemplado. En primer lugar, el periódico permite mantener el contacto con la asignatura una vez se ha acabado el periodo lectivo, lo que es especialmente beneficioso para los alumnos que tienen que acudir a la convocatoria de septiembre, como ocurre con Comunicación oraly escrita en lengua española I, el periódico de los estudiantes de COELEI y COEEI del Centro Asociado de Madrid - Gregorio Marañón.

En segunda instancia, la publicación digital contribuye a la actualización continua en la materia. Aunque los estudiantes hayan finalizado ya las asignaturas del primer cuatrimestre, pueden mantener el interés por estar actualizados y seguir aprendiendo el resto del curso. Y hay quien incluso se ha animado a crear su propio periódico en Paper.li.

\section{Imagen 6. estadísticas de Paper.li de los tres periódicos analizados}

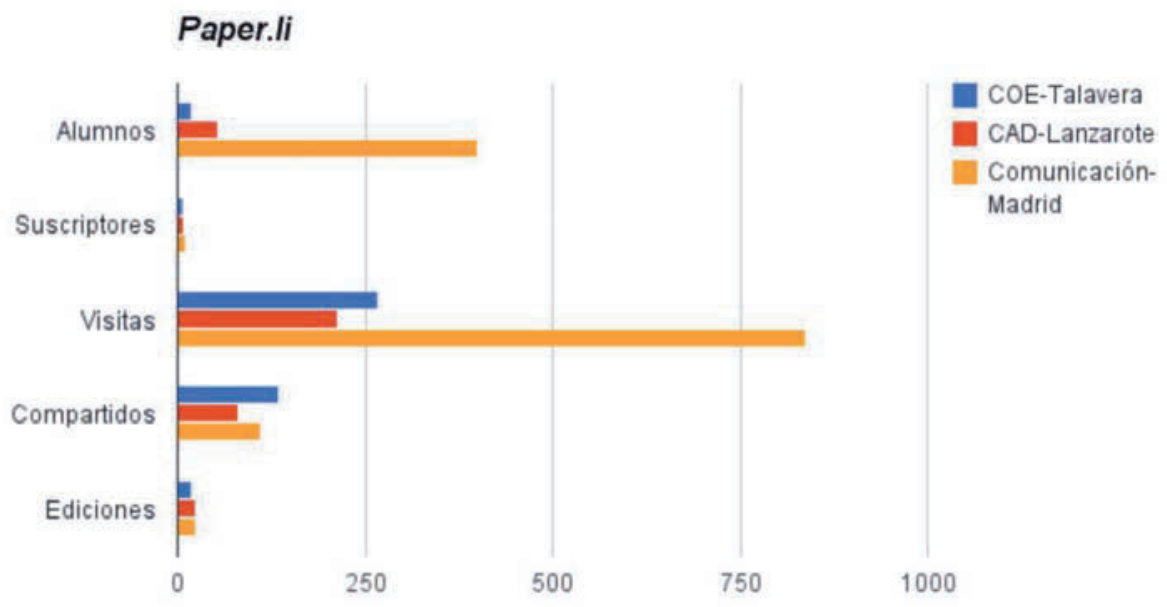




\section{A MOdo DE CONCLUSIÓN}

El uso de una herramienta como Paper.li permite al docente y al alumnado desarrollar una serie de competencias, capacidades, estrategias y destrezas propias de la era digital.

La forma habitual de comunicación y socialización a través de las redes sociales o el microblogging tienden a ser mensajes breves y concisos, donde la capacidad de síntesis en la expresión escrita es una competencia deseable.

Es bien sabido que un usuario de la Web 2.0 recibe tal cantidad ingente de información que si no sabe gestionarla adecuadamente puede producirle la sensación de no poder abarcarla ni asimilarla, a esto se le llama infoxicación. Para evitar esta frustración hay que saber discriminar la información relevante y fiable de la que no es en la lectura en formato digital. Es necesario desarrollar la comprensión lectora y determinadas estrategias de lectura interrelacionadas, como son el skimming o 'barrida del texto' y el scannig o 'búsqueda de información específica'.

Entre los motivos que suelen esgrimir tanto el profesorado como el alumnado para no usar las herramientas de la Web 2.0, suele ser el aumento de la carga de trabajo. También puede ser el recelo a utilizar herramientas cuyo uso no está implantado. Por otro lado, puede suceder que el desánimo sea propiciado por el desconocimiento. En otras ocasiones, se puede conocer la herramienta, pero llevarla al ámbito educativo de modo eficaz no es tarea fácil.

El empleo de herramientas innovadoras normalmente queda relegado a actividades optativas o complementarias que, a pesar de que no puntúan al alumno ni son remuneradas económicamente para el docente, aportan gran satisfacción y ayudan a fortalecer las relaciones interpersonales del grupo-clase. En el caso de Paper.li es recomendable que la publicación del periódico sea semanal y en ningún caso diaria, así como limitar el número de fuentes para no saturar con demasiada información y en lugar de ser una actividad motivante resulte distractora.

Consideramos que es motivador para el estudiante de la UNED implicarlo en su proceso de aprendizaje, hacerlo partícipe, autónomo y crítico a partir del trabajo colaborativo. Casi sin darse cuenta, el alumnado estará desarrollando destrezas de comprensión lectora y de expresión escrita, fomentando el diálogo ante una audiencia real que no se limitará al docente y a los compañeros, de nuevo en situaciones auténticas de comunicación.

El mayor potencial que ofrece Paper.li, tal y como apunta Araujo (2012), es que permite una comunicación real y significativa. En definitiva, creemos que el potencial educativo de esta herramienta es enorme en una sociedad en la que las TIC están ya integradas y donde es necesario desarrollar competencias digitales para desenvolverse con soltura en el ámbito académico y profesional que nos espera y que ya está aquí. 


\section{Bibliografía}

Araujo, J. C. (2012) «Creación de periódicos en línea con Paper.li como recurso didáctico para el aprendizaje de lenguas». Revista Electrónica de Tecnología Educativa, 42. Recuperado el 10/02/2015 de bttp:/ / edutec.rediris.es/Revelec2/Revelec42/pdf/Edutec-e_n42-Arujo.pdf

Mancera, A. (2011) ¿Cómo se "babla" en los cibermedios? El español coloquial en el periodismo digital. Berna: Peter Lang.

Toro, G. (2010) «Usos de Twitter en la Educación Superion». Serie Bibliotecología y Gestión de la Información, $n^{\circ}$ 53. Chile: Universidad Tecnológica Metropolitana. Recuperado el 10/02/2015 de bttp://eprints.rclis.org/14190/1/Serie_N\%C2\%BA_53_Enero_2010_Uso_de_Twitter_en_la_Educaci\% C3\%B3n_Superior.pdf

Torres, D. y Delgado, E. (2009) «Estrategia para mejorar la difusión de los resultados de investigación con la web 2.0». El Profesional de la Información, 19, nº 5, pp. 534-539. Recuperado el 10/02/2015 de http:/ / eprints.rclis.org/13901/

carriazo@flog.uned.es

elifernandez@talavera.uned.es

marnayan@usal.es 